\title{
The Coupled Oscillator Model of Between-Hand Coordination in Alternate-Hand Tapping: A Reappraisal
}

\author{
Andras Semjen \\ Centre National de la Recherche Scientifique
}

\author{
Richard B. Ivry \\ University of California, Berkeley
}

\begin{abstract}
Single and alternating hand tapping were compared to test the hypothesis that coordination during rhythmic movements is mediated by the control of specific time intervals. In Experiment 1, an auditory metronome was used to indicate a set of timing patterns in which a 1 -s interval was divided into 2 subintervals. Performance, measured in terms of the deviation from the target patterns and variability, was similar under conditions in which the finger taps were made with 1 hand or alternated between the 2 hands. In Experiment 2, the modality of the metronome (auditory or visual) was found to influence the manner in which the produced intervals deviated from the target patterns. These results challenge the notion that bimanual coordination emerges from coupling constraints intrinsic to the 2 -hand system. They are in accord with a framework that emphasizes the control of specific time intervals to form a series of well-defined motor events.
\end{abstract}

Bimanual coordination during sustained periodic movements has been a major topic in the research on human motor control. Previous work has shown that the two hands act in an interdependent way in the sense that the spatial, temporal, or intensive characteristics of the movements on each hand are constrained with respect to the movements on the other hand. Under the dynamic systems approach to movement control, the coordinated unfolding of movement trajectories has been modeled in terms of coupled oscillators (e.g., Haken, Kelso, \& Bunz 1985; Kelso, Holt, Rubin, \& Kugler, 1981; Yamanishi, Kawato, \& Suzuki, 1980). Central to this approach is the notion of stability. Stable coordination is characterized by the fact that the phase delay between the oscillating limbs is close to a target value and that the variability of the phase delay is small. Conversely, unstable coordination exhibits systematic distortion with respect to the intended phase delay as well as an increase in the variability of the observed phase delay.

Extensive experimental work has demonstrated that the coordination between two body segments (e.g., the two hands) is stable at slow or moderate movement frequencies when they oscillate in-phase or antiphase. In the in-phase mode, the limbs are at the same points of their movement cycles at the same time, whereas in

Andras Semjen, Centre de Recherche en Neurosciences Cognitives (CRNC), Centre National de la Recherche Scientifique (CNRS), Marseille, France; Richard B. Ivry, Department of Psychology, University of California, Berkeley.

We thank W. Byblow, D. Vorberg, and three anonymous reviewers for helpful comments on an earlier version of this article. Preparation of this article was partially supported by National Institutes of Health Grants NS30256 and NS33504.

Correspondence concerning this article should be addressed to Andras Semjen, CRNC-CNRS, 31 Chemin Joseph Aiguier, 13402 Marseille Cedex 20, France, or to Richard B. Ivry, Department of Psychology, University of California, Berkeley, California 94720 . Electronic mail may be sent to semjen@lnf.cnrs-mrs.fr or to ivry@socrates.berkeley.edu. the antiphase mode, the limbs are in opposite points of their movement cycles at the same time. For example, simultaneous rotations of the wrists in the transverse plane are considered in-phase if the movements mirror each other, with both hands moving simultaneously toward or away from the body midline. The coordination mode is said to be antiphase when one hand is moving toward the body midline while the other is moving away. Likewise, tapping in synchrony with both index fingers would involve in-phase coordination, whereas tapping in a regularly alternating manner would involve antiphase coordination. Phase delays that deviate from in-phase or antiphase relations typically give rise to less stable or unstable hand coordination in repetitive cyclic movements (e.g., Kelso, 1984; Zanone \& Kelso, 1997).

From the coupled oscillator framework, the stability of coordination is taken to reveal the interactions between the component oscillators. Thus, it emphasizes intrinsic constraints on hand coordination. One impressive achievement of this approach is its capacity to provide a compact mathematical description of the conditions under which the stability of coordination can be maintained or disrupted (Haken et al., 1985). This form of modeling is not generally constrained by the psychological or neurophysiological processes underlying these interactions.

In place of the psychological and neural entities ... traditionally proposed to understand coordination and control, the dynamical approach posits dynamical structures of control spread across several (neural, metabolic, biomechanical, informational, and environmental) levels of analysis and whose functioning is bound by dynamical principles of self-organization. (Schmidt \& Fitzpatrick, 1996, p. 199)

Nonetheless, there exist a number of questions that remain unanswered within this framework and that have received minimal attention. One of the most important among these questions concerns the way an intended (or actual) phase delay is perceived and controlled. Beyond the possibility that the concurrently unfolding movement trajectories may be compared continuously with each other, two solutions can be considered. People may check whether 
some specific anchor points on each trajectory coincide with each other in a predetermined way. For example, intermittent crossreferencing of the movement trajectories might assess if the two limbs reach maximum extension or flexion at the same time during in-phase coordination or if one limb reaches maximum extension and the other reaches maximum flexion at the same time during antiphase coordination. Alternatively, people may set the time intervals that separate adjacent anchor points within or across trajectories. Salient anchor points (such as maximum flexion or extension) or well-determined motor events (such as finger taps) could mark the boundaries of the successive time intervals. For instance, antiphase coordination would involve setting the occurrence of a finger tap performed by one hand halfway in the time period determined by two successive finger taps performed with the other hand. Under this view, hand coordination would be organized by the limbs complying with (timing) constraints extrinsic to the two-hand system.

One model which makes the assumption that the temporal distribution of serial motor acts is governed by an internal timekeeper is the two-level timing model of Wing and Kristofferson (1973). This model was developed to account for unimanual tasks but was extended subsequently to synchronic bimanual tapping and alternate tapping with both hands. For instance, during bimanual, in-phase tapping, the coordination of the hands would be achieved by sending a common timing signal to both hands to synchronize their movements (Vorberg \& Hambuch, 1978, 1984; see also Helmuth \& Ivry, 1996). For antiphase tapping, hand coordination would be achieved by directing the signals of a single timekeeper in alternation to the right and left hand (Wing, Church, \& Gentner, 1989).

The purpose of this article is to further substantiate the notion that the coordination between the hands may be powerfully patterned through the control of specific time intervals that separate the occurrence of well-defined motor events associated with each hand's trajectory. From this perspective, we want to show that the phasing between the hands may depend, at least in part, on the participant's capacity to generate the required timing patterns. To this end, we first analyze the timing requirements of the bimanual finger-tapping task used by Yamanishi et al. (1980). Our analysis of the Yamanishi et al. task leads us to the expectation that the main results observed in the bimanual condition should be reproducible in a single-hand tapping task involving similar timing requirements. Next, we report experimental data that support this contention. Finally, we discuss the implications of these results for current models of bimanual timing and coordination.

\section{Yamanishi et al.'s (1980) Bimanual Tapping Task and Coupled Oscillator Models}

Our focus on the Yamanishi et al. (1980) study is motivated by historical and methodological concerns. Historically, the study represents one of the first attempts to introduce the coupled oscillator model into the field of hand coordination. Methodologically, the study is noteworthy in that the authors used a semicontinuous tapping task. Almost all subsequent studies conducted in the coupled oscillator framework have used tasks that involve continuous free oscillations of the limbs.

Yamanishi et al. (1980) had participants tap in synchrony with two visual pacing signals, one for the left hand and the other for the right hand. For each visual metronome, the period of the interstimulus interval (ISI) was fixed at $1,000 \mathrm{~ms}$. The main experimental variable was the phase delay $\phi$ between the occurrence of the left and right pacing signals. The value of $\phi$ was randomly varied across trials from 0 to $900 \mathrm{~ms}$ in steps of $100 \mathrm{~ms}$. When expressed as a fraction of the metronome period, these values correspond to phase delays ranging between 0.1 and 0.9 . After 10 response cycles were completed with a given phase delay, the visual signals were extinguished and the participants continued tapping the same pattern for 20 additional cycles. When correctly performed, the task involved the production of sequences of 1,000-ms within-hand intervals, with one hand leading the other hand by a time interval defined by $\phi$. Results showed that this time interval (i.e., the phase delay) was closest to the required value and exhibited minimum variability for synchronous bimanual tapping $(\phi=0)$ and for regular alternate-hand tapping $(\phi=0.5)$. Other phase relationships were produced with higher variability and often showed systematic distortions in the direction of the nearest stable phase delay. For instance, when the target phase was 0.4 or 0.6 , distortions toward the 0.5 phase value could be observed. That is, the participants' tapping shifted toward regular alternation between the hands.

From these results, Yamanishi et al. (1980) modeled hand coordination in terms of coupled oscillators. The model assumes that two oscillators control the movements, one associated with the movements of the left hand and one associated with the movements of the right hand. According to the theory, the oscillators are prone to mutual perturbations or interference: Bilateral interactions between the right and left oscillator change their phase relation. The model shows that these perturbations are minimal at $\phi$ values of 0 and 0.5 and that they increase with other $\phi$ values, entailing less stable coordination between the hands. Subsequent dynamic theories of hand coordination used more sophisticated coupling functions in describing the greater stability of hand coordination in synchronic and alternating movements (e.g., Haken et al., 1985). In their review of Yamanishi et al. (1980) and subsequent replications (e.g., Tuller \& Kelso, 1989), Schöner and Kelso (1988) argued that the stability of coordination as a function of relative phase is intrinsically determined. However, the intrinsic coordination tendencies can be modulated by extrinsic constraints such as information coming from the environment (e.g., the metronome signals) or higher level cognitive processes (e.g., memory). Coordination can be expected to be more stable when the intrinsic tendencies and the task requirement coincide (cooperation) than when they do not coincide (competition). Although this model and that proposed by Yamanishi et al. (1980) differ with respect to the processes whereby coordination may be perturbed, both share the notion that the main characteristics of hand coordination arise from constraints intrinsic to the two-hand collective.

\section{Timing Control Hypothesis}

Consider an alternative description of the task that emphasizes the rhythmic patterns produced by the series of finger taps (see Figure 1). When tapping in the in-phase mode, $\phi=0$, a simple pattern is created of evenly paced intervals with a period of 1,000 ms. For all other phases, the response cycle produced by the repetitive movements of one hand is subdivided by the taps of the other hand. The simplest subdivision is achieved when $\phi=0.5$ 


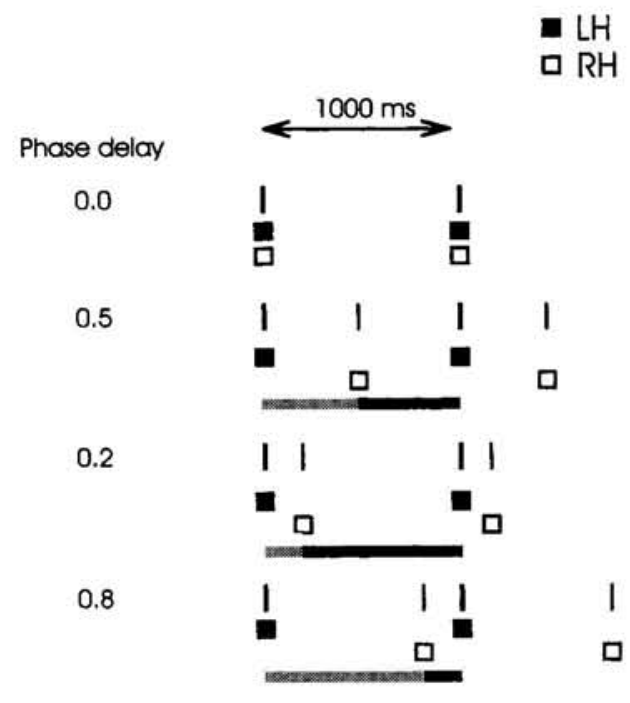

Figure 1. Main features of the timing requirements of the bimanual tapping task. Metronome events are represented by vertical bars. Finger taps are represented by black boxes (left hand; $\mathrm{LH}$ ) and white boxes (right hand; RH). The response cycles of both hands are either concurrent ( $\phi=0.0$, with simultaneous tap onsets) or shifted by some proportion of the period duration ( $\phi$ values of $0.5,0.2$, and 0.8 are shown). Within-hand response cycle duration is measured between tap onsets of the same hand (i.e., time intervals delimited by black and white boxes, respectively). When both hands' response cycles are shifted against each other, the taps of the right hand (white boxes) subdivide the response cycle of the left hand (black boxes) into equal (with $\phi=0.5$ ) or unequal (short-long or long-short) subintervals. The first subinterval (gray horizontal bars) is a measure of the actual phase delay between the hands.

because, in this case, the two subintervals are of equal duration$500 \mathrm{~ms}$. With all other phases, the subintervals are of unequal duration. To give an example, we show in Figure 1 the interresponse intervals (IRIs) generated across hands (see gray and black horizontal bars) with $\phi=0.2$ or 0.8 . These subdivisions create a salient short-long or long-short timing pattern. This analysis leads us to consider the possibility that in performing the Yamanishi et al. (1980) task, the participants controlled the coordination between the hands by complying with the timing constraints inherent to the production of groups of rhythmic taps. We refer to this hypothesis as the timing control hypothesis.

The timing control hypothesis focuses on an external constraint in the sense that the rhythmic patterns are not linked to the two-hand collective. As such, this hypothesis would predict that the coordination patterns observed in the bimanual tapping task of Yamanishi et al. (1980) would also be obtained in an analogous task performed with other pairs of effectors (e.g., two fingers of the same hand) or, perhaps more interesting to note, when the task is performed with a single effector. If the coordination constraints observed during two-hand tapping are due to the representation of the timing patterns associated with the formation of rhythmic events, one should observe similar constraints across a range of conditions regardless of the effectors involved.

Summers, Bell, and Burns (1989) obtained results that were consistent with this hypothesis. These authors had participants imitate rhythmic tone sequences in three response conditions: alternate-hand tapping with the index fingers of both hands, tapping in alternation with the index and middle fingers of the same hand, and tapping with the index finger of a single hand. The total cycle time was $1,200 \mathrm{~ms}$ with different conditions created by varying the asynchrony between two adjacent tones to set subinterval ratios of $11: 1,5: 1,3: 1,2: 1,1.4: 1$, and $1: 1$. These ratios correspond to $\phi$ values ranging between 0.08 and 0.50 . The results showed almost identical performance under the three response conditions. Poorest reproduction was observed when the subintervals formed high-order ratios $(11: 1,5: 1)$; these patterns were distorted toward lower order ratios. The best reproduction of the target rhythm was obtained with the $1: 1$ ratio $(\phi=0.5)$. Moreover, with the $1.4: 1$ ratio $(\phi \approx 0.42)$, attraction toward the $2: 1$ pattern rather than to the $1: 1$ pattern was observed.

The above results are in line with those from numerous studies on the dynamic organization of rhythmic groups. This literature has consistently shown that people can precisely reproduce auditory patterns composed of intervals forming low-order integer ratio relations such as $1: 1$ or $2: 1$. Interval patterns that do not obey these ratios-those that require more complex rhythms-are often distorted (when tapped out with the same finger) such that the produced ratio is shifted toward simpler ratios (i.e., lower level ratios, integer ratios, or both; e.g., Essens, 1986; Essens \& Povel, 1985; Fraisse, 1956, 1982; Povel, 1981). The distortion is less pronounced when the rhythmic pattern can be mapped onto a metrical representation (Essens \& Povel, 1985; Povel, 1981). In a metrical representation, the time intervals of the rhythmic pattern are generated as multiples of a timing unit or base duration. The processes actually involved may be described in terms of a clockcounter model in which a single timekeeper provides a base duration and in which a counter mechanism counts multiples of the base duration in order to generate the target intervals (see Collier \& Wright, 1995). Under this hypothesis, higher order ratio intervals may appear more difficult to perform than low-order ratio intervals owing to limitations in the counting process. In contrast, noninteger ratio intervals may prove more difficult to produce than integer ratio intervals owing to complexities in identifying the base timing unit. However, the use of a metrical grid for rhythm representation is not mandatory. People can encode and reproduce time intervals forming complex (Collier \& Wright, 1995) or even simple (Essens \& Povel, 1985) ratios in a nonmetrical fashion, eventually at the expense of less precise and less accurate reproduction.

The timing control hypothesis posits that, when bimanual tapping is not performed in a synchronized manner, controlling the between-hand time intervals is a fundamental goal for processes involved in controlling coordination. Thus, coordination should be stable if the timing pattern is easy to produce such as one involving a low-order integer ratio, whereas it should be unstable if the timing pattern does not lend itself to such a representation. A central prediction of the timing control hypothesis is that the timing patterns performed during alternate-hand tapping should be quite similar to those one would observe in an analogous singlehand tapping task. As noted above, Summers et al. (1989) provided initial support for this conjecture. In the first experiment reported below, we replicate and extend this work. We adopted the procedure of Yamanishi et al. (1980), using the same basic response period $(1,000 \mathrm{~ms})$ and range of phase delays (from 0 to 0.9 by steps of 0.1 ). Our focus was on a comparison between when the rhythms 
were produced by tapping with the index fingers of both hands (two-hand task) and when the rhythms were produced by tapping with the index finger of a single hand (one-hand task). ${ }^{1}$

With phase delays different from zero $(\phi>0)$, the task involves the subdivision of the response cycle into two subintervals forming either high-order integer ratio relations $(9: 1,4: 1)$, low-order noninteger ratio relations $(2.3: 1,1.5: 1)$, or low-order integer ratio relations $(1: 1)$. The timing control hypothesis makes similar predictions for the two-hand and one-hand tasks concerning the duration and variability of the subintervals, as well as the variability of the overall cycle duration. Assuming that the subintervals are controlled in a serial fashion, close correspondence between the required and performed subinterval duration would only be expected with the phase delay $\phi=0.5$ because this condition involved a low-order integer ratio relation $(1: 1)$ between the subintervals. $^{2}$ This condition corresponds to isochronous one-hand tapping or regular alternate-hand tapping. Note that the coupled oscillator model also predicts the stability of this condition during two-handed tapping. With phase delays that involve high-order $(9: 1,4: 1)$ integer ratio relations (i.e., $\phi=0.1,0.2,0.8,0.9)$, distortions toward lower order ratio relations could be expected to occur, by means of lengthening of the shorter subinterval and shortening of the longer subinterval (see Essens \& Povel, 1985; Fraisse, 1956). In contrast, from the coupled oscillator model, one would predict that distortions should be consistently toward the nearest stable coordination mode (i.e., $\phi=0$ or synchronized tapping). As such, the prediction is that the shorter interval would be shortened and the longer interval would be lengthened. Finally, with noninteger $(2.3: 1,1.5: 1)$ ratio relations between the subintervals (i.e., $\phi=0.3,0.4,0.6,0.7$ ), distortions toward neighboring ( $2: 1$ or $1: 1)$ integer ratio relations are expected. Here again, the two hypotheses make different predictions. The timing control hypothesis would predict distortions toward either a $2: 1$ or $1: 1$ pattern; the coupled oscillator model designates only the $1: 1$ relation $(\phi=0.5)$ as a natural attractor.

Next, consider the expected variability of the subintervals. Weber's law has been found to hold for both the production and perception of time intervals (e.g., Allan, 1979, 1992; Getty, 1975; Ivry \& Hazeltine, 1995; Killeen, 1992), although violations are frequently found for very short intervals (e.g., Peters, 1989). Hence, the timing control hypothesis predicts that in both the two-hand and one-hand tasks, a roughly linear relationship should be observed between the standard deviation of the subintervals and the mean of the subintervals. In contrast, the coupled oscillator model for two-hand tapping predicts smaller variability for $\phi=0.5$ (antiphase tapping) than for any other phase delay greater than zero. Moreover, the timing control hypothesis leads to an additional prediction regarding the standard deviation of the cycle duration (target $=1,000 \mathrm{~ms}$ ). When the response cycle is produced as the sum of two shorter subintervals $(\phi>0)$, its standard deviation should be less than the standard deviation of the same response cycle when produced without any subdivision $(\phi=0)$. This reduction should be greatest when the subintervals are equal in duration ( $\phi=0.5$; see Appendix). A reduction in the standard deviation of the cycle duration following subdivision has been observed in numerous studies (Deutsch, 1983; Semjen, Vorberg, \& Schulze, 1992; Walter, Corcos, \& Swinnen, 1998; see also Killeen, 1992). In these studies, the subdivision always created intervals that were integer multiples of one another. However, other factors may add to the observed variability of the cycle duration if the target subintervals are not related in that manner.

To this point, we have assumed that the subintervals of the response cycle were controlled in a serial fashion (by a single timekeeper plus a counting mechanism or by two concatenated timekeepers). Alternatively, the timing of the cycle duration and the timing of the first subinterval could be controlled by two hierarchically organized timekeepers that are started in common points in time (Vorberg \& Hambuch, 1978, 1984). One test to decide whether the time intervals in a sequence of events are controlled on several levels (hierarchic model) or a single level (serial model) involves the analysis of the standard deviation of the time intervals marked off by taps that have identical position in the successive cycles. In the present case, the comparison would bear on response cycles measured between odd-numbered taps or evennumbered taps. The mean cycle duration is the same no matter what tap starts the cycle. In contrast, the standard deviation of the cycle duration would differentiate between the alternative versions of these timing control models. If the concatenation model holds, the standard deviation should be the same whatever the starting tap. If the hierarchical model holds, the standard deviation should be less for the starting tap that coincides with the output of the higher order timer (Vorberg \& Hambuch, 1984). Note that the hierarchical model does not predict any decrease of the cycle standard deviation when the phase delay is different from zero because by this model, the cycle duration does not emerge as the sum of two shorter subintervals. In addition, the hierarchical model is mute about possible distortions of the mean subintervals at different required phase delays.

In summary, the first experiment was aimed at substantiating the notion that timing control is the core aspect of hand coordination in alternate-hand tapping. To this end, we adopted the Yamanishi et al. (1980) tapping task and compared performance between two-hand and one-hand tapping conditions. In previous replications of the Yamanishi et al. study, data were only obtained during the synchronization phase with the inducing stimuli (Tuller \& Kelso, 1989) or during imitation of the rhythmic sequence in the absence of the metronome (Summers et al., 1989). In the present experiment, we collected and analyzed the data obtained during both the synchronization and continuation phases.

\section{Experiment 1}

\section{Method}

\section{Participants}

Six persons (including Andras Semjen), 3 men and 3 women participated in the experiment. All of the participants had practiced a musical instrument for at least 8 years.

\footnotetext{
${ }^{1}$ The $\phi=0$ condition cannot be reproduced in single-hand tapping. This condition was therefore replaced by regular tapping without any subdivision of the basic period.

${ }^{2}$ We do not make any strong assumption about whether the timing control operates with a metrical representation of the rhythmic patterns (which would involve a single timekeeper and a counter mechanism) or a nonmetrical representation (which would involve two concatenated timekeepers, except for $\phi=0.5$, when a single timekeeper is sufficient).
} 


\section{Apparatus and Measurements}

The response keys consisted of circular touch plates mounted on a wooden support fixed to a table. The participant was seated with his or her forearms lying on the table and the index fingers placed above the response keys. The response keys were interfaced to a Compaq Prolinea PC. All onsets and offsets of contact between the finger and the response key were detected. IRIs were measured to the nearest millisecond between successive contact onsets. The computer also controlled a sound card (DREAM XPC-16; Dream S. A., Semur, France) that produced the periodic pacing tones. The tones were presented through a monitor speaker (Roland MA20; Roland Taiwan Electronic Music Corporation, Taiwan) placed approximately $1 \mathrm{~m}$ in front of the participant above the video monitor. The taps on the response plate were clearly audible.

\section{Task and Design}

The experiment consisted of two sessions of $1.5 \mathrm{hr}$ each, separated by 1-7 days. The sessions differed in the task performed, with one session devoted to two-hand tapping and the other to one-hand tapping. Half of the participants started with the two-hand task, and the other half started with the one-hand task. In both tasks, the participants were requested to tap periodically with their index finger (or fingers) on the response key (or keys), first in synchrony with the sounds of the metronome (synchronization) and then without the metronome (continuation).

Two-hand task. In this task, the participant had to synchronize a left-hand tap with a high-pitched $(988 \mathrm{~Hz})$ tone of the metronome and a right-hand tap with a low-pitched $(740 \mathrm{~Hz})$ tone of the metronome. Tone duration was $40 \mathrm{~ms}$ and the ISI between successive high-pitched tones and successive low-pitched tones was $1,000 \mathrm{~ms}$. The asynchrony (or phase delay, $\phi$ ) between the high-pitched and low-pitched tones was varied across trials from $0 \mathrm{~ms}$ to $900 \mathrm{~ms}$, in steps of $100 \mathrm{~ms}$. For $\phi=0$, only low-pitched tones were presented. In this condition, the participant had to synchronize the responses of both hands to the same metronome sound. Before each trial, a message was displayed on the video screen, informing the participant about the phase delay for the forthcoming trial. The trial was initiated when the participant touched a specific response plate. The message was then erased and the metronome tones commenced. Participants could listen to the metronome until they were ready to respond at the required tempo and phase delay. They were required to initiate each response sequence with a left-hand tap synchronized to a high-pitched tone. After 40 response cycles, the metronome was turned off and the participant continued to tap the same pattern for another 40 cycles. Participants were told to maintain the tapping pattern induced during synchronization throughout the continuation phase. Four blocks of 10 correct trials were recorded during the session. Within each block of trials, each phase delay (from 0 to $900 \mathrm{~ms}$ ) was presented once, in a random order.

One-hand task. In this task, the metronome delivered the same stimulus patterns as in the two-hand task. That is, for each trial, high- and low-pitched tones were presented recurrently with $1,000-\mathrm{ms}$ ISI, and the asynchrony between the high- and low-pitched tones was manipulated to create 10 possible phase delays. Again, only the low-pitched tone was presented for $\phi=0$. The participant's task was to synchronize a tap with the right index finger with each tone of the sequence during 40 response cycles and to continue the same pattern of tapping for an additional unpaced 40 cycles. Response sequences were to be initiated with a tap synchronized to a high-pitched tone. All other aspects of the procedure were the same as in the two-hand task.

On-line control of data acquisition. The tap sequences were controlled on-line to detect extra taps (bounces), tap omissions, or incorrect assignment of the taps to tones at the beginning of the series. The trial was interrupted and immediately repeated whenever any of these events were detected.

\section{Data Reduction and Analysis}

The mean and variance of the IRIs were calculated trial by trial, with these measures obtained separately for the synchronization and continuation phases. The first five response cycles from each phase were excluded from the data sets. Individual scores for each phase delay condition were calculated by averaging these statistics over trials. We describe the results in terms of response cycles and subintervals (see Figure 1). Response cycles are (a) within-hand IRIs in the two-hand task and (b) IRIs measured between successive odd-numbered taps and successive even-numbered taps in the one-hand task. Subintervals are (a) between-hand IRIs in the two-hand task and (b) IRIs measured between successive taps in the one-hand task. In the two-hand task, we arbitrarily assigned the left-hand response cycle to serve as the reference interval. Given this, the first subinterval (represented in Figure 1 by gray horizontal bars) occurs between a left-hand tap and the next right-hand tap. The second, complementary subinterval occurs between this right-hand tap and the next lefthand tap. Analogously, the first subinterval in the one-hand task occurs between an odd-numbered tap and the immediately following tap, whereas the second subinterval occurs between an even-numbered tap and the immediately following tap. Note that during synchronization, the beginning of the first subinterval was marked off by the high-pitched tone and the beginning of the second subinterval was marked off by the low-pitched tone.

\section{Results}

\section{Subintervals}

Duration. The mean difference between the required and observed duration of the first subinterval is given in Figure 2A. The deviation from the required value was close to zero when the response cycle was subdivided into equal subintervals $(\phi=0.5$ ) by either taps of the same hand (one-hand task) or taps of the alternating hands (two-hand task). For all other phase delays, there were systematic distortions. In general, the shorter of the subintervals was lengthened, and the longer was shortened. One exception was for $\phi=0.4$ or 0.6 . With these values, the direction of distortions was reversed. As Figure $2 \mathrm{~A}$ shows, the pattern was very similar for the one-hand and two-hand task. An analysis of variance (ANOVA) was performed on the difference between observed and required first subinterval duration to test the effects of task (one hand, two hand), metronome (presence, absence; i.e., synchronization, continuation), and phase delay. Only phase delay proved to have a significant effect, $F(8,40)=66.49, p<.001$. A significant Task $\times$ Phase Delay interaction, $F(8,40)=4.49, p<$ .001 , pointed to differences between one-hand and two-hand tapping at extreme values of $\phi(0.1$ and 0.9$)$. When the analysis was restricted to $\phi$ values between 0.2 and 0.8 , the Task $\times$ Phase Delay interaction was not reliable, $F(6,30)=1.61$. A significant Metronome $\times$ Phase Delay interaction, $F(8,40)=2.69, p<.05$, was also observed. This finding indicated differences in the magnitude of distortion in subinterval duration during synchronization as compared with continuation, although the basic pattern is quite similar for the two phases.

Subinterval ratios. As a consequence of the deviations in the duration of the subintervals, the ratio of the subintervals systematically departed from the target values with all $\phi$ values other than 0.5 . Required and observed interval ratios (long/short) are given in Figure 2B. The observed ratios were almost identical for the one-hand and two-hand tasks except for $\phi=0.1$ and 0.9 . With $\phi$ values of $0.3,0.4,0.6$, and 0.7 , the distortions in subinterval 

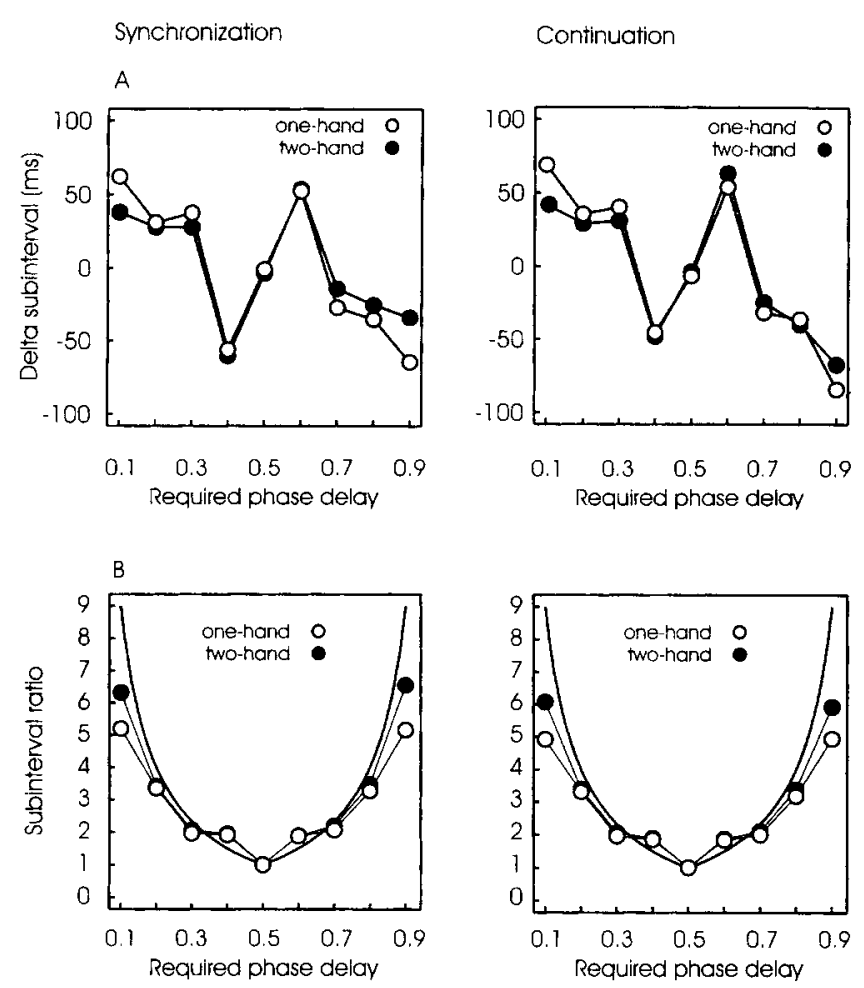

Figure 2. Subinterval duration and subinterval ratio in Experiment 1. Panel A: Mean difference between observed and required duration of the first subinterval (delta subinterval), as a function of required phase delay, in the one-hand and two-hand task during synchronization and continuation. Panel B: Mean of the observed ratios between the long and short subintervals, as a function of phase delay, in the one-hand and two-hand task during synchronization and continuation. The required ratios appear on the thick U-shaped line.

duration established mean interval ratios close to 2 . Note that the production of identical interval ratios here required distortions of the short and long subintervals in the opposite directions for the $\phi=0.3$ and 0.7 conditions compared with the $\phi=0.4$ and 0.6 conditions. With $\phi$ values of 0.2 and 0.8 , the interval ratios scattered around 3.3 (instead of the required value of 4). Finally, with $\phi$ values of 0.1 and 0.9 , the mean interval ratio was close to 5 in the single-hand task and close to 6 in the two-hand task (instead of 9).This difference was probably linked with the fact that the short subinterval underwent greater lengthening in the single-hand task than in the two-hand task, owing perhaps to limitations in how quickly people can tap a single finger in succession.

In sum, the observed interval ratios had two characteristic features in both tasks: preference for the $1: 1$ and 2:1 ratios and shifts toward lower order ratios when the required ratios were high. To provide a sense of whether these results reflect the averaging of different distortion patterns across participants, we categorized the individual long/short interval ratio data by assigning them to their closest integer ratio. The distribution of the individual ratios is shown in Table 1 as a function of phase delay. Note that in this presentation, the ratios obtained with complementary $\phi$ values (e.g., 0.1 and 0.9 ) were pooled under a common entry. The ratios observed for synchronization and continuation were also lumped together. Therefore, the number of observations is $N=24$ for the entries with complementary $\phi$ values, and $N=12$ with $\phi=0.5$. Table 1 shows considerable variability between the participants in the range $\phi<0.3$ and $\phi>0.7$, indicating that the mean values shown in Figure $2 \mathrm{~B}$ are somewhat misleading. However, essentially no scatter is observed for data in the range $0.3 \leq \phi \leq 0.7$.

Variability. The mean of the standard deviation and the coefficient of variation of the first subinterval are given in Figure 3. The values on the abscissa correspond to the mean observed subintervals rather than to the target subintervals. Figure $3 \mathrm{~A}$ shows that standard deviation increased monotonically for IRIs ranging between 300 and $900 \mathrm{~ms}$. Correspondingly, the coefficient-ofvariation function (Figure 3B) shows a rapid initial decrease over the shortest subintervals before stabilizing at a value just below $5 \%$ for subinterval durations of $500 \mathrm{~ms}$ or more. Despite somewhat higher coefficient-of-variation values observed for the two-hand task in the short subinterval range, the pattern of results was qualitatively the same across task and metronome conditions. Linear regression of the mean standard deviation to the mean IRI in the $500-\mathrm{ms}$ to $900-\mathrm{ms}$ range indicated a slightly steeper slope and better fit for the one-hand task (slope $=0.05, r^{2}=98 \%$ ) than for the two-hand task (slope $=0.04, r^{2}=84 \%$ ).

\section{Response Cycle}

Duration. During synchronization, the average cycle duration was equal to the ISI of the metronome-1,000 ms. During continuation, there were small deviations from the target cycle duration at certain phase delays (Table 2). Note that the largest overshoots and undershoots occurred in both tasks at the same phase delays ( 0.4 and 0.6 for overshoots and 0.1 and 0.9 for undershoot). An ANOVA performed on the individual mean cycle duration yielded a significant effect for phase delay, $F(9,45)=2.28, p<$ .03. The effect of task (one-hand, two-hand) and the Phase Delay $\times$ Task Interaction were not significant $(F<1)$.

Variability. The average standard deviation of the cycle duration is given in Figure $4 \mathrm{~A}$ as a function of task (one hand vs. two

Table 1

Distribution of the Individual Long:Short Interval Ratios in Experiment 1

\begin{tabular}{|c|c|c|c|c|c|c|c|c|c|}
\hline \multirow[b]{2}{*}{$\phi$ and task } & \multicolumn{9}{|c|}{ Interval ratio } \\
\hline & $9: 1$ & $8: 1$ & $7: 1$ & $6: 1$ & $5: 1$ & $4: 1$ & $3: 1$ & $2: 1$ & $1: 1$ \\
\hline \multicolumn{10}{|l|}{$0.1 \& 0.9$} \\
\hline One hand & & & & 7 & 14 & 3 & & & \\
\hline Two hand & & 2 & 9 & 8 & 3 & 2 & & & \\
\hline \multicolumn{10}{|l|}{$0.2 \& 0.8$} \\
\hline One hand & & & & & & 6 & 18 & & \\
\hline Two hand & & & & & & 12 & 12 & & \\
\hline \multicolumn{10}{|l|}{$0.3 \& 0.7$} \\
\hline One hand & & & & & & & & 24 & \\
\hline Two hand & & & & & & & & 24 & \\
\hline \multicolumn{10}{|l|}{$0.4 \& 0.6$} \\
\hline One hand & & & & & & & & 24 & \\
\hline Two hand & & & & & & & & 24 & \\
\hline \multicolumn{10}{|l|}{0.5} \\
\hline One hand & & & & & & & & & 12 \\
\hline Two hand & & & & & & & & & 12 \\
\hline
\end{tabular}

Note. Each observed ratio was assigned to the closest integer ratio. 
Synchronization
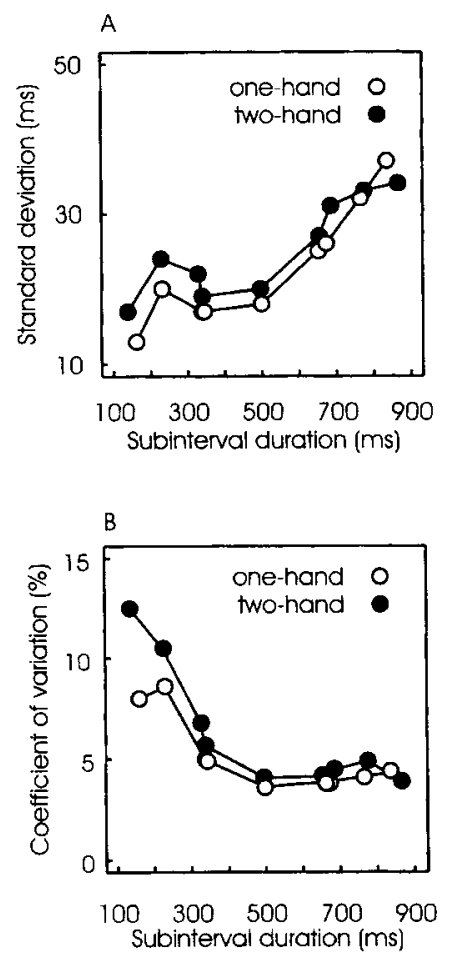

Continuation
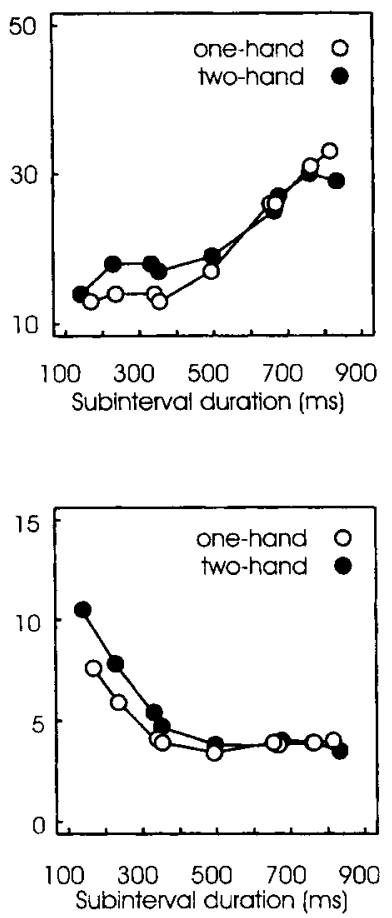

Figure 3. Subinterval variability in Experiment 1. Mean standard deviation (Panel A) and mean coefficient of variation (Panel B) of the first subinterval as a function of its mean duration in the one-hand and two-hand task during synchronization and continuation.

hand). Without any subdivision of the response cycle $(\phi=0)$, the standard deviation was less in the two-hand task than in the one-hand task, $F(1,5)=41.74, p<.01$. This finding is consistent with results reported by Helmuth and Ivry (1996). When the response cycle was subdivided into equal-duration subintervals $(\phi=0.5)$, the standard deviation dropped to a minimum, as expected. Across the range of $\phi$ values from 0.1 to 0.5 , standard deviation generally decreased; for values ranging from 0.5 to 0.9 ,

Table 2

Average Cycle Duration (in Milliseconds) During Continuation in the One-Hand and Two-Hand Task, as a Function of Required Phase Delay, in Experiment 1

\begin{tabular}{ccc}
\hline & \multicolumn{2}{c}{ Task } \\
\cline { 2 - 3 } Phase delay & One hand & Two hand \\
\hline 0.0 & 1,008 & 1,008 \\
0.1 & 986 & 978 \\
0.2 & 1,006 & 999 \\
0.3 & 1,005 & 1,013 \\
0.4 & 1,012 & 1,020 \\
0.5 & 989 & 996 \\
0.6 & 1,009 & 1,017 \\
0.7 & 1,002 & 995 \\
0.8 & 1,006 & 990 \\
0.9 & 984 & 979 \\
\hline
\end{tabular}

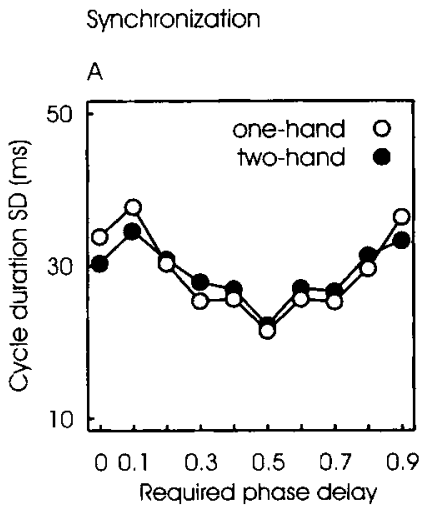

Continuation
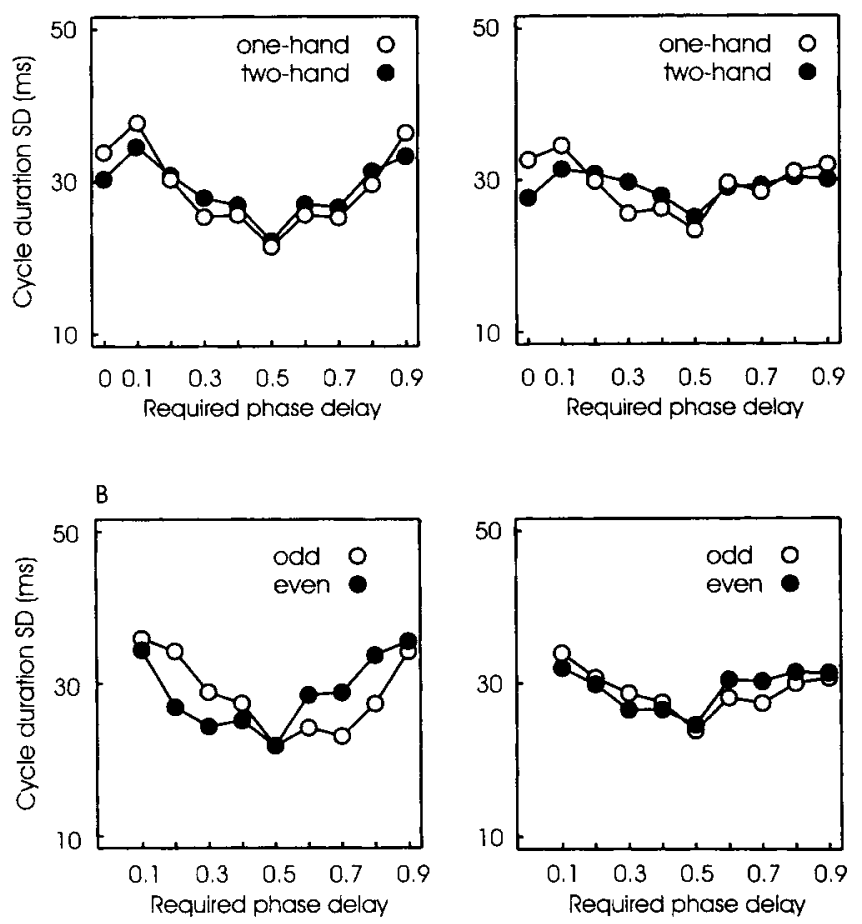

Figure 4. Standard deviation of the cycle duration in Experiment 1. Panel A: Mean standard deviation, as a function of the required phase delay, in the one-hand and two-hand task during synchronization and continuation. The cycle durations measured between odd-numbered and even-numbered taps (one-hand task) or the cycle durations for left-hand and right-hand taps (two-hand task) were collapsed. Panel B: Mean standard deviation of the duration of the response cycles delimited by odd-numbered and evennumbered taps, as a function of required phase delay, during synchronization and continuation.

standard deviation increased in a symmetric fashion. Of greatest interest in the present study, this pattern was essentially identical in the one-hand task and two-hand task.

The standard deviation for the response cycles marked off by odd-numbered taps and even-numbered taps is given in Figure 4B, averaged over the one-hand and two-hand tasks. With phase delays between 0 and 0.5 , the standard deviation was higher for cycles that started from odd-numbered taps, whereas with phase delays between 0.5 and 0.9 , the standard deviation was higher for cycles that started from even-numbered taps. The crossover occurred at $\phi=0.5$. Thus, the standard deviation was higher when the cycle was measured across taps initiating the shorter of the two subintervals. These effects were more pronounced during synchronization than during continuation.

An ANOVA was performed on the individual standard deviation scores to test the effects of task, metronome, cycle delimiter (even-numbered taps, odd-numbered taps), and phase delay. Only the main effect of phase delay was found to be significant, $F(8$, $40)=16.23, p<.001$. There was a Task $\times$ Phase Delay interaction, $F(8,40)=2.49, p<.05$, due to the fact that at the extreme values of $\phi(0.1$ and 0.9$)$, the standard deviation in the one-hand 
task was higher than in the two-hand task, whereas at all other $\phi$ values, the one-hand task provided slightly smaller standarddeviation values. In a second ANOVA restricted to $\phi$ values between 0.2 and 0.8 , this interaction proved to be statistically nonsignificant. No other interactions involving the one-hand versus two-hand contrasts were significant. The significant three-way interaction between cycle delimiter, phase delay, and metronome conditions, $F(8,40)=13.22, p<.001$, indicated that the standarddeviation differences linked with cycle delimiter were essentially confined to the synchronization phase.

\section{Discussion}

The results of this experiment support the hypothesis that timing control is the core aspect of hand coordination in periodic alternate-hand tapping. On the basis of the timing control hypothesis, we anticipated that the results for the one- and two-hand tasks would be similar on a set of a priori criteria. These included predictions concerning the duration of the subintervals created within the basic tapping cycle by taps of the same hand (one-hand task) or of the other hand (two-hand task), the variability of the subintervals, and the variability of the cycle duration. Despite small discrepancies observed mainly at the extreme phase values $(0.1$ and 0.9$)$, the pattern of results showed impressive concordance across the one- and two-hand tasks on all three criteria.

The duration of the subintervals, and hence, the phase delay in the two-hand task, exhibited systematic distortions in the direction predicted by the timing control hypothesis. Higher order interval ratios were distorted toward lower order ratios, and low-level noninteger ratios were distorted in the direction of low-level integer ratios. As noted earlier, simple time interval ratios (1:1 and $2: 1$ ) constitute stable timing patterns in that they are reproduced more precisely than higher order or more complex ratios. Our results differ from those reported in Yamanishi et al. (1980) when the target phase values were 0.4 or 0.6 . In the earlier report, the observed distortions were toward the antiphase hand coordination mode $(\phi=0.5)$. This finding prompted Yamanishi et al. to consider the antiphase mode as a stable state of coordination toward which other, less stable modes of coordination are attracted. Because antiphase coordination involves the generation of between-hand subintervals with a ratio of 1, Yamanishi et al.'s finding can also be accounted for in terms of shift toward a more attractive pattern of timing. In the present study, we did not observe an attraction toward the antiphase mode in the two-hand task for $\phi=0.4$ or 0.6 . Instead, these phase delays were distorted toward $\phi=0.33$ or $\phi=0.66$, respectively-phase delays in which the between-hand subintervals have a ratio of $2: 1$ (see also Summers et al., 1989). Because this pattern was also observed in the one-hand task, these shifts in the between-hand phase delays can be viewed as resulting from an attraction toward a more stable timing pattern. Note again that these phase delays are not considered naturally attractive under the coupled oscillator modeling of between-hand coordination.

The timing control hypothesis predicts that the standard deviation of the subintervals will increase with their mean, whereas the coupled oscillator hypothesis predicts that the variability of phase delay will be lower for $\phi=0.50$ than for any other nonzero phase delay. We observed the former pattern in both the two-hand and one-hand tasks for subintervals longer than $300 \mathrm{~ms}$. Moreover, the function relating the standard deviation to the mean was approximately linear in the 500-900-ms range, consistent with Weber's law in the temporal domain. However, there is a sharp rise in the coefficient of variation for intervals below $300 \mathrm{~ms}$. Other investigators have observed a similar rise at very short tapping intervals (e.g., Peters, 1989). This apparent breakdown of Weber's law might reflect the fact that for short intervals, the variability contributed by duration-dependent (timekeeper) processes is relatively small compared with that contributed by duration-independent (motor) processes. Alternatively, the rise here might reflect instability in performance when attempting to produce temporal patterns with high ratios between the subintervals.

One distinguishing feature of the timing control hypothesis is the prediction that the variability $(S D)$ of the cycle duration will decrease when the response cycle is produced as the sum of two subintervals. As expected, the greatest decrease was observed when the subintervals were equal in duration $(\phi=0.50)$. Fractionated production of the response cycle entailed some reduction in the standard deviation for other phase delays as well, although the extreme $\phi$ values were actually accompanied by an increase, rather than a decrease, in the cycle standard deviation. The summation hypothesis assumes that the subintervals are controlled in a strictly serial fashion with no interaction between successive subintervals and with no drift over the extent of the trial. The high variability for patterns requiring high subinterval ratios may indicate that for these conditions, the subdivision process may introduce a new source of variability. Alternatively, as noted above, this increase may result from the fact that the participants had difficulty in establishing a stable pattern for these conditions (see Table 1).

Some comment is in order concerning the relationship between the cycle standard deviation and cycle delimiters. When the response cycle was subdivided into equal subintervals, the cycle standard deviation did not depend on how the cycles were measured (e.g., between odd-numbered or even-numbered taps). In contrast, when the response cycle was subdivided into unequal subintervals, the cycle standard deviation was higher when the cycles were measured between the taps that initiated the shorter subintervals rather than between the taps that terminated the shorter subintervals (or, equivalently, initiated the longer subintervals). This effect was reliable only during the synchronization phase. Variations in the response cycle standard deviation as a function of the cycle delimiter can be expected to occur under a hierarchical model of timing in which the cycle with the lower standard deviation is controlled by a higher order timekeeper, and the first subinterval within this cycle is controlled by a subordinate timekeeper (Vorberg \& Hambuch, 1978, 1984). However, a timing model of this kind would not easily predict the observed subinterval distortions and the decrease in the cycle standard deviation with nonzero phase delays. Moreover, it is difficult to see why hierarchical control would be limited to the synchronization phase. One can suppose, as a more plausible explanation, that during the synchronization phase, the participants corrected for the synchronization error mainly by adjusting the timing of the tap that initiated the shorter subinterval. The relatively higher standard deviation of the response cycles measured between these taps may be a consequence of this process because correction for synchronization error is known to enhance the variability of the IRIs (Vorberg \& Wing, 1996). 


\section{Experiment 2}

The results of Experiment 1 corroborate the main predictions of the timing control hypothesis. This hypothesis provides a different emphasis on the processes underlying bimanual coordination during periodic movements than the coupled oscillator model introduced by Yamanishi et al. (1980). The timing control hypothesis emphasizes extrinsic constraints involving the representation of the temporal relationships between the subintervals. These constraints are relevant not only to two-handed tasks such as those used by Yamanishi et al. but also to the one-handed task used in Experiment 1.

What remains unclear is why the participants in the Yamanishi et al. study (1980) were attracted toward the 1:1 timing pattern (antiphase tapping), whereas the participants in the present study, as well as those of Summers et al. (1989), preferred to shift toward the 2:1 timing pattern? One methodological difference between the studies involves the stimulus modality used to entrain performance. Yamanishi et al. used a visual metronome. In the current study and in that of Summers et al., the target intervals were presented in the auditory modality. With an auditory metronome, the participants could clearly perceive that the intertone intervals were unequal for all phase delays different from 0.0 or 0.5 . Given this, they would know that they must produce unequal betweenhand subintervals to meet the task demands except in the $\phi=0.5$ condition. In contrast, the visual metronome may not have provided sufficiently sharp discrimination between the subintervals when they were close in duration (e.g., with $\phi=0.4$ or 0.6 ), a hypothesis supported by the time perception literature indicating poorer temporal acuity in vision than audition (see review in Allan, 1979). Thus, the attraction toward the antiphase coordination mode in these conditions may have matched the participants' perception of the temporal pattern. We tested this hypothesis in Experiment 2.

\section{Method \\ Participants \\ Six female participants volunteered in the experiment. Only one of them had played a musical instrument for more than 5 years.}

\section{Task}

The experiment consisted of two sessions of $1.5 \mathrm{hr}$ each, separated by 1-7 days. Both sessions involved two-hand tapping. The sessions differed in the modality used to establish the target phase. In the first session, visual stimuli were used for the pacing metronome. In the second session, auditory stimuli were used for the metronome. A fixed order of these conditions was adopted because we were concerned that the participants might develop a rhythmic response pattern in the auditory condition and maintain this strategy during the visual condition. In particular, they might discover that an alternating strategy was only viable on a small percentage of the trials. Note that our main interest in Experiment 2 was whether the 1:1 pattern was more likely to occur when performance was guided by a visual metronome. Thus, the main data of interest were collected prior to any possible contamination from the auditory condition.

The task and procedure in the auditory session were identical to those used in the two-hand task of Experiment 1. For the visual session, the stimulus display consisted of three light-emitting diodes (LEDs): a central fixation LED and two lateralized LEDs positioned $5^{\circ}$ of visual angle to the right or left of the fixation marker. Participants were told to synchronize a left-hand tap with the left-side LED and a right-hand tap with the right-side
LED. Each LED was periodically illuminated for $20 \mathrm{~ms}$. The ISI between successive illuminations of each LED-that is, the cycle durationwas $1,000 \mathrm{~ms}$. As in Experiment 1, the asynchrony between both LEDs was varied across trials from $0 \mathrm{~ms}$ to $900 \mathrm{~ms}$ in steps of $100 \mathrm{~ms}$. For $\phi=0$, both LEDs were illuminated simultaneously, and the participants had to synchronize the responses of both hands to the LEDs. For $\phi>0$, the participants were required to initiate each response sequence with a lefthand tap synchronized to a left-side stimulus. Before each trial, a message was displayed on the video screen indicating whether the LEDs would blink simultaneously or in succession during the forthcoming trial. Participants could observe the metronome signals until they were ready to respond at the required tempo and phase delay. All other aspects of the trials were the same as in Experiment 1.

\section{Results}

\section{Subintervals}

Subinterval duration. The mean difference between the required and observed duration of the first subinterval is given in Figure 5A. The subintervals were closest to the required value in both tasks with $\phi=0.5$. In general, the short subinterval $(\phi<0.4)$ tended to be lengthened and the long subinterval $(\phi>0.6)$ tended to be shortened. These effects were slightly stronger in the visual task. Of greatest interest in this experiment, the visual and auditory tasks led to opposite distortions with $\phi$ values of 0.4 or 0.6 . In the visual task, the participants tended to produce equal subintervals
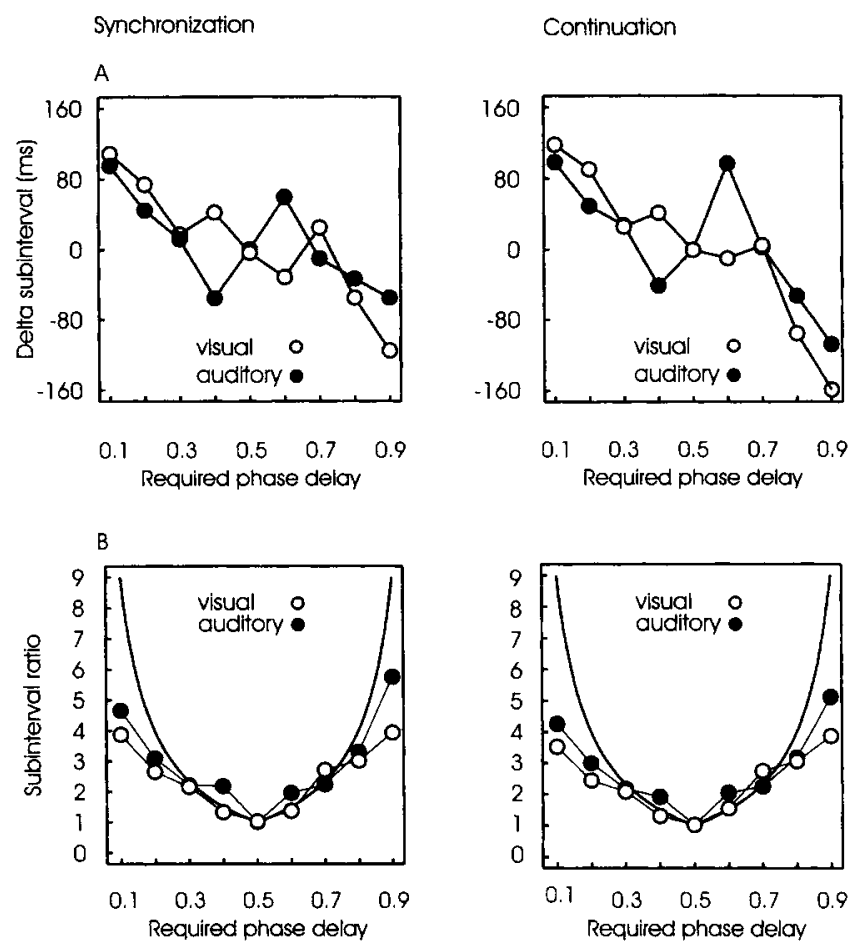

Figure 5. Subinterval duration and subinterval ratio in Experiment 2. Panel A: Mean difference between observed and required duration of the first subinterval (delta subinterval), as a function of required phase delay, in the visual and auditory task during synchronization and continuation. Panel B: Mean of the observed ratios between the long and short subintervals, as a function of phase delay in the visual and auditory task, during synchronization and continuation. The required ratios appear on the thick $U$-shaped line. 
for these conditions, similar to those reported by Yamanishi et al. (1980). Thus, with $\phi=0.4$, the first subinterval was longer than required, and with $\phi=0.6$, it was shorter. The reverse trend was found in the auditory task. Here, with $\phi=0.4$, the first subinterval was shorter than required, and with $\phi=0.6$, it was longer. The results for the auditory condition replicate those observed in Experiment 1.

The difference scores were subjected to an ANOVA that tested the effects of task, metronome, and phase delay. Only phase delay had a significant effect, $F(8,40)=23.27, p<.001$. A significant Task $\times$ Phase Delay interaction, $F(8,40)=8.01, p<.001$, confirmed the contrasting profile of subinterval distortions for the visual and auditory tasks. Given our predictions regarding the difference between the two modalities, a separate test was performed on the interaction between task (visual vs. auditory) and a restricted set of phase delays $(0.4,0.5$, and 0.6$)$. This interaction was also significant, $F(2,10)=11.51, p<.01$. The Metronome $\times$ Phase Delay interaction, $F(8,40)=6.12, p<.001$, was also significant, owing to the fact that the subintervals were more distorted during continuation at the extreme phase delays.

Subinterval ratios. Required and observed subinterval ratios (long/short) are given in Figure 5B. Table 3 presents the distribution of the ratios from individual trials into the nine integer categories, with the values calculated across the synchronization and continuation phases as in Experiment 1. The ratio data provide further confirmation of the modality effect for $\phi$ values of 0.4 and 0.6. The mean value for the visual task is closer to a ratio of 1 , consistent with the hypothesis that the participants perceived the two subintervals as equal in duration. Indeed, as Table 3 shows, most trials were assigned to the 1:1 category. In contrast, in the auditory task, all of the interval ratios were assigned to the 2:1 category, and the mean subinterval ratio was 1.96 .

For the other values of $\phi$, the subinterval ratio results were similar to those observed in Experiment 1. The 1:1 ratio $(\phi=0.5)$ was precisely reproduced in both tasks, and high-level ratios tended to be replaced by lower level ratios. A modality effect is

Table 3

Distribution of the Individual Long:Short Interval Ratios in Experiment 2

\begin{tabular}{|c|c|c|c|c|c|c|c|c|c|}
\hline \multirow[b]{2}{*}{$\phi$ and task } & \multicolumn{9}{|c|}{ Interval ratio } \\
\hline & $9: 1$ & $8: 1$ & $7: 1$ & $6: 1$ & $5: 1$ & $4: 1$ & $3: 1$ & $2: 1$ & $1: 1$ \\
\hline \multicolumn{10}{|l|}{$0.1 \& 0.9$} \\
\hline Visual & & & & 2 & 3 & 10 & 8 & 1 & \\
\hline Auditory & & & 3 & 5 & 7 & 7 & & 2 & \\
\hline \multicolumn{10}{|l|}{$0.2 \& 0.8$} \\
\hline Visual & & & & & & 2 & 13 & 9 & \\
\hline Auditory & & & & & & 6 & 18 & & \\
\hline \multicolumn{10}{|l|}{$0.3 \& 0.7$} \\
\hline Visual & & & & & & 2 & 6 & 16 & \\
\hline Auditory & & & & & & & 3 & 21 & \\
\hline \multicolumn{10}{|l|}{$0.4 \& 0.6$} \\
\hline Visual & & & & & & & & 7 & 17 \\
\hline Auditory & & & & & & & & 24 & \\
\hline \multicolumn{10}{|l|}{0.5} \\
\hline Visual & & & & & & & & & 12 \\
\hline Auditory & & & & & & & & & 12 \\
\hline
\end{tabular}

Note. Each observed ratio was assigned to the closest integer ratio. also evident at the extreme phase delays, and the compression effect for the auditory condition appears to be more pronounced than in Experiment 1.

Subinterval variability. The mean of the standard deviation and the coefficient of variation of the first subinterval are presented in Figure 6. The abscissa values are based on the observed subinterval durations rather than the target durations. The standard deviation was greater in the visual task than in the auditory task, $F(1,5)=16.43, p<.01$. It is interesting to note that this effect appears to extend into the continuation phase, although the auditory advantage is greatly attenuated during this phase. The relationship between the standard deviation and the mean interval during synchronization with the visual metronome was more irregular. The increase in the standard deviation at $\phi$ values of 0.4 and 0.6 is reminiscent of the standard-deviation data reported in Yamanishi et al.'s (1980) study. Linear regression of the mean standard deviation to the mean IRI in the 500-ms-900-ms range for the continuation data revealed a slightly steeper slope for the visual task (slope $=0.08, r^{2}=99 \%$ ) than for the auditory task (slope $=0.06, r^{2}=87 \%$ ), supporting the hypothesis that temporal acuity is poorer for vision than audition.

As in Experiment 1, the coefficient of variation was highest for the shortest subintervals and decreased to a relatively stable value for subinterval durations of $500 \mathrm{~ms}$ or more. The asymptote in the auditory task was slightly above $5 \%$, whereas in Experiment 1 , it was slightly below this value. This difference is most probably related to the fact that all the participants of the first experiment were musically trained, whereas those of the second experiment were not.

\section{Response cycle}

Duration. During synchronization, the average cycle duration was equal to the ISI of $1,000 \mathrm{~ms}$. During continuation, the participants consistently undershot the target duration at the two shortest and two longest phase delays, with this deviation greater in the visual task (Table 4). Overshoots occurred only in the auditory task at phase delays of $0.3,0.4,0.6$, and 0.7. An ANOVA performed on the individual mean cycle duration yielded a significant effect only for phase delay, $F(9,45)=7.59, p<.001$.

Variability. The average standard deviation of the cycle duration is given in Figure $7 \mathrm{~A}$ as a function of task (visual vs. auditory). In both tasks and during both synchronization and continuation, the standard deviation tended to decrease for $\phi$ values ranging between 0.1 and 0.5 and to increase for $\phi$ values ranging between 0.5 and 0.9 . This pattern reproduces that observed in Experiment 1. Thus, the standard deviation dropped to a minimum in both tasks for $\phi=0.5$, that is, with regular alternating tapping of both hands (the antiphase coordination). The standard deviation for response cycles delimited by odd-numbered taps and even-numbered taps is given in Figure 7B. During synchronization, the standard deviation was higher for cycles that started from odd-numbered taps when the phase delay was less than 0.5 , whereas the standard deviation was higher for cycles that started from even-numbered taps when the phase delay was greater than 0.5. The odd- and even-numbered functions cross over at $\phi=0.5$.

An ANOVA performed on the standard deviation scores tested the effects of task, metronome, cycle delimiter, and phase delay 

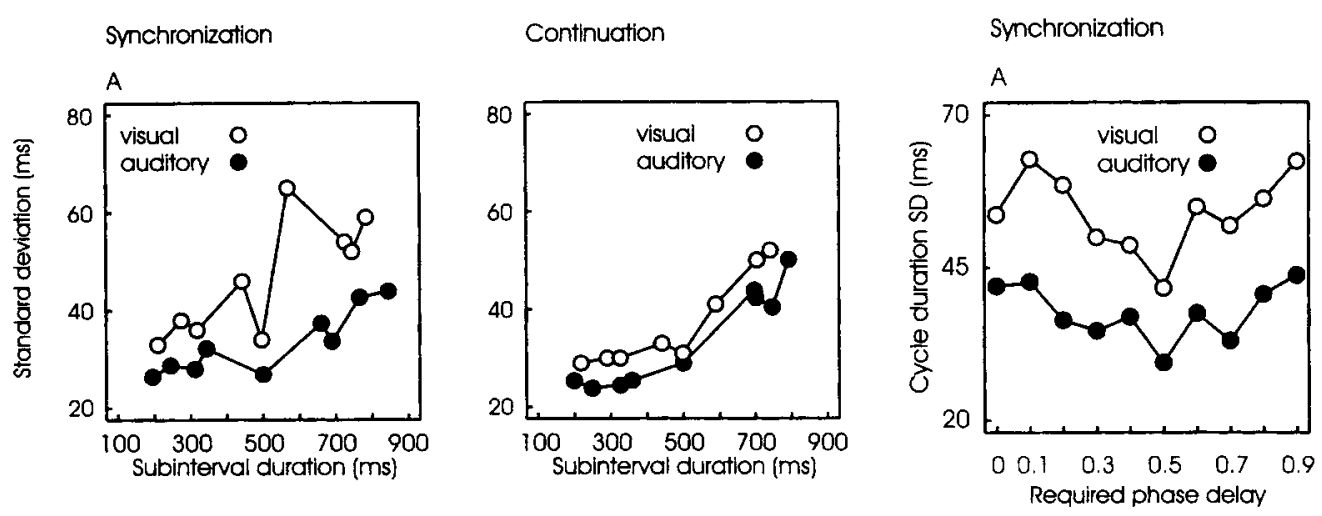

\section{Continuation}
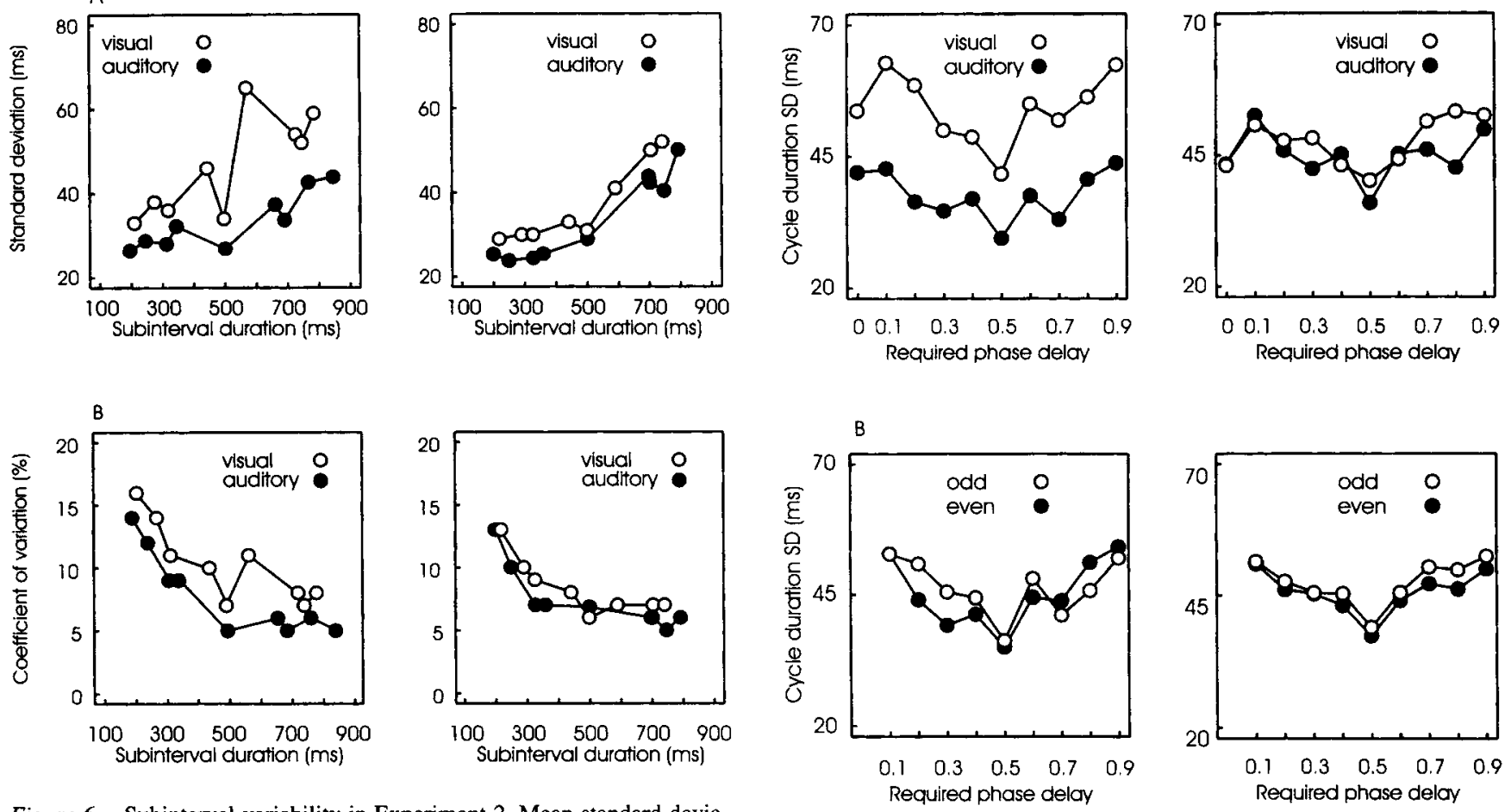

Figure 6. Subinterval variability in Experiment 2. Mean standard deviation (Panel A) and mean coefficient of variation (Panel B) of the first subinterval, as a function of its mean duration in the visual and auditory task, during synchronization and continuation.

$(0.1$ to 0.9$)$. The effects of phase delay, $F(8,40)=9.70, p<.001$, and cycle delimiter, $F(1,5)=7.44, p<.05$, were found to be significant. A significant Task $\times$ Metronome interaction, $F(1$, $5)=49.49, p<.001$, indicated that the average standard deviation during synchronization was higher in the visual task than in the auditory task, whereas during continuation, the standard deviation was similar across tasks. A significant three-way interaction between metronome, cycle delimiter, and phase delay confirmed that the effects of cycle delimiter seen during synchronization were

Table 4

Average Cycle Duration (in Milliseconds) During Continuation in Visual and Auditory Task, as a Function of Required Phase Delay, in Experiment 2

\begin{tabular}{crr}
\hline & \multicolumn{2}{c}{ Task } \\
\cline { 2 - 3 } Phase delay & Visual & Auditory \\
\hline 0.0 & 962 & 994 \\
0.1 & 958 & 971 \\
0.2 & 983 & 993 \\
0.3 & 1,000 & 1,034 \\
0.4 & 995 & 1,058 \\
0.5 & 991 & 998 \\
0.6 & 989 & 1,039 \\
0.7 & 970 & 1,014 \\
0.8 & 944 & 982 \\
0.9 & 939 & 951 \\
\hline
\end{tabular}

Figure 7. Standard deviation of the cycle duration in Experiment 2. Panel A: Mean standard deviation, as a function of the required phase delay, in the visual and auditory task during synchronization and continuation. The cycle durations measured for left-hand and right-hand taps were collapsed. Panel B: Mean standard deviation of the duration of the response cycles delimited by odd-numbered (left hand) and even-numbered (right hand) taps, as a function of required phase delay, during synchronization and continuation.

eliminated during continuation, $F(4,80)=4.68, p<.001$. The standard deviation of the cycle duration in the auditory condition was higher in this experiment than in Experiment 1 (see Figures 4 and 7). This discrepancy points again to group differences in musical expertise.

\section{Discussion}

In Experiment 2, either a visual or auditory metronome indicated the required timing pattern for alternate-hand tapping. With a visual metronome, we replicated the experimental arrangement under which Yamanishi et al. (1980) and Tuller and Kelso (1989) observed phase shifts toward the antiphase pattern $(\phi=0.5)$ for required phase delays of 0.4 or 0.6 . With an auditory metronome, we replicated the experimental arrangement under which, with the same required phase delays, phase shifts toward 0.33 and 0.66 were observed (Summers et al., 1989, and Experiment 1 of the present study). The main aim of the experiment was to show that the direction of phase shift was conditional upon the modality of the inducing metronome. With a visual metronome, we expected a shift toward 1:1 subinterval ratios $(\phi=0.5)$, presumably owing to the fact that the participants would tend to perceive the subinter- 
vals as equal in length. In contrast, we expected that the duration difference between the subintervals would be easily perceived in the auditory condition, and thus the produced distortion would be shifted to a simple ratio of $2: 1$.

The results are in accord with these predictions. Note, however, that the auditory metronome induced a more consistent behavior across participants than the visual metronome at $\phi$ values of 0.4 and 0.6 . In the visual condition, some of the shifts occurred in the $2: 1$ direction rather than in the $1: 1$ direction. In the auditory case, no trials were suggestive of a shift in the 1:1 direction. Yamanishi et al. (1980) also reported large discrepancies between the participants with respect to the direction of the distortion of the phase delay. Although these authors did not present analyses in terms of subinterval ratio, their graphs clearly show that at certain required phase delays (e.g., $\phi=0.7$ ), some participants performed a $2: 1$ ratio, whereas for other participants, the produced ratio was approximately $2.7: 1$. From the present results, it thus appears that the sensory modality of the entraining metronome may critically influence the selection of the attractive pattern. Therefore, the notion that the preferred timing pattern (or coordination mode) is uniquely determined by coupling influences intrinsic to the hand collective must be qualified.

A systematic relationship between cycle delimiters and cycle duration standard deviation occurred during the synchronization phase, as in Experiment 1. This result is consistent with the hypothesis that the difference between the two delimiters is due to the effects of synchronization error correction, rather than to the effects of a hierarchical form of timing control. Further study is required to determine why error correction may occur in an asymmetric fashion when the subintervals are unequal.

Comparisons between the visual and auditory conditions revealed greater overall timing variability in the visual task. While it is possible that this reflects a practice effect because all participants performed the visual task during the first session, we suspect that the difference reflects an advantage for temporal processing of auditory signals. First, the difference in timing variability (as indexed by the standard deviation of the cycle duration and of the subintervals) was greatest during synchronization, although it persisted in attenuated form during continuation (see also, Kolers \& Brewster, 1985). A practice effect would be expected to also remain during continuation. Second, the observed asymmetry is consistent with previous modality effects in the literature (see Allan, 1979).

What remains to be seen is whether the modality effect is manifest in timing processes per se (see Penney, Gibbon, \& Meck, 2000 ) or whether it arises from other sources of variability. Synchronization requires the contribution of corrective mechanisms that keep the motor responses locked to the metronome events. It is often assumed that the input to the corrective mechanisms is the time asynchrony between the occurrence of the taps and the metronome events (Semjen, Schulze, \& Vorberg, 2000; Vorberg \& Wing, 1996). The enhanced timing variability during synchronization with the visual metronome might be due to less precise input to the corrective loop.

Despite such differences, similar patterns were obtained in the visual and auditory conditions in terms of the relationship between the required phase delay and cycle duration standard deviation, including minimal standard deviation in the $\phi=0.5$ condition. This suggests that the internal timing mechanisms had the same functional characteristics whether they were invoked using the visual or the auditory metronome.

\section{General Discussion}

Two contrasting views of bimanual coordination were examined in this study. One view asserts that the main characteristics of hand coordination in repetitive cyclic tasks arise from dynamic interactions (coupling) between biological oscillators, that is, from constraints intrinsic to the two-hand system (e.g., Kelso et al., 1981; Schöner \& Kelso, 1988; Yamanishi et al., 1980). Studies on the coordination of (low- or medium-frequency) bimanual movements have consistently emphasized that two coordination patterns, inphase and antiphase, are intrinsically stable and behave as attractor states (Haken et al., 1985; Kelso, 1984; Yamanishi et al., 1980). Other forms of phasing patterns can be learned with practice, although even here, the target phase may only be manifest at certain points within the cycle (e.g., Zanone \& Kelso, 1997).

The alternative view we propose postulates that the main constraints associated with the production of repetitive movements arise from the internal representation of the temporal requirements for the task. To reflect this emphasis, we have termed this view the timing control hypothesis. For bimanual tapping, the temporal representation specifies the time intervals that are produced by successive taps across the hands, as well as the overall cycle duration for successive taps within each hand (or other salient events within the movement trajectory). The stability of a repetitive pattern will be a function of the temporal relationship between the subintervals. In particular, patterns that lend themselves to simple rhythmic relationships will be more stable than those that require complex rhythms (or are arhythmic). Under the timing control hypothesis, the emphasis is on constraints that are extrinsic to the two-hand system. Indeed, such constraints are not limited to bimanual coordination. As shown in Experiment 1, they are also manifest during the production of repetitive unimanual movements and play an important role in how we perceive periodic events. As demonstrated in Experiment 2, the temporal organization of bimanual movements is intimately linked to how the participants perceive the task (see also Klapp et al., 1985; Summers et al., 1989).

An essential component of the timing control hypothesis is the idea that the observed temporal pattern reflects the operation of a central timekeeper (e.g., Wing \& Kristofferson, 1973). This process is, at least at an abstract level, amodal in nature (Fraisse, 1963; Ivry \& Hazeltine, 1995; Ivry \& Keele, 1989; Keele, Pokorny, Corcos, \& Ivry, 1985; Rosenbaum \& Patashnik, 1980). Our focus in this article has been to assess the importance of central timekeeping rather than to characterize the nature of this process. Nonetheless, the observed timing patterns conformed to quantitative predictions derived from the hypothesis that the intervals were controlled by a central timekeeper (or timekeepers) in a serial fashion (Vorberg \& Wing, 1996). First, a Weber-type relationship was observed between the standard deviation of the subintervals and their means (Ivry \& Hazeltine, 1995; Killeen \& Weiss, 1987), although violations were apparent for the shortest intervals. Second, in general, the standard deviation of the cycle duration decreased when this period was produced as the sum of two subintervals, especially when the subintervals were equal in duration. 
Third, the distortion of the subintervals was consistently toward simple ratios such as $2: 1$.

The latter result is especially important in contrasting the timing control hypothesis with the coupled oscillator model. The coupled oscillator view would predict a strong attraction to the antiphase mode for target phase relations near 0.5. However, when an auditory metronome was used to define the task, the distortions for the 0.4 and 0.6 conditions were in a direction away from 0.5 . We assume that the participants perceived the inequality between the subintervals and distorted the target pattern to the closest simple rhythm.

It is important to note that the coupled oscillator model has proven useful in accounting for aspects of coordination beyond those observed during bimanual movements. The basic ideas have been used to examine coordination between the upper and lower limbs (Baldissera, Cavallari, \& Civaschi, 1982; Kelso \& Jeka, 1992) as well as segments of a single limb (Kelso, Buchanan, \& Wallace, 1991). The framework has also been generalized to capture single-limb coordination. For example, the interactions between two individuals (Schmidt, Carello, \& Turvey, 1990) or between an individual and periodic external signals (Wimmers, Beek, \& van Wieringen, 1992) have been modeled in terms of coupled oscillators. A strength of this approach has been that a common abstract account based on the dynamics of coupled oscillators can provide a description of the interactions observed under these various conditions. We expect that the generality here reflects, at least in part, the constraints associated with representing temporal relationships between different events, regardless of whether the events derive from the actions of one hand or of two hands of a single individual or from the actions produced by two individuals.

All of the movements in our experiments were performed at a comfortable speed. In contrast, much of the work based on the coupled oscillator framework has focused on how patterns of stability vary as a function of tempo. In particular, the model has provided an elegant description of the phase transition that occurs at a critical frequency such that antiphase movements become unstable and participants experience an unintended transition to an in-phase mode. Such transitions are observed across a wide range of conditions, emphasizing the fundamental dynamic principles captured by a coupled oscillator model. Phase transitions are not only observed with multilimb movements but also arise between single limb movements and external events. For example, Wimmers et al. (1992) observed a phase transition from antiphase to in-phase mode when participants tracked a moving visual stimulus. They suggested that the transition occurs "because at a particular frequency in the antiphase mode an informational resolution ceiling is reached that can be resolved by changing to the in-phase mode" (Wimmers et al., 1992, p. 225). This view would seem consistent with the timing control hypothesis. Coordination between the two events requires sufficient resolution of the temporal information characterizing each component of the task.

More generally, this idea might provide an account of phase transitions within the timing control hypothesis. Antiphase movements require not only a representation of the target intervals but also a means for resolving the asymmetric gestures for the two hands. The information load is reduced during in-phase movements given the symmetry of the gestures. At this point, we only intend this conjecture to provide a qualitative description of how phase transitions may reflect limitations on how information is represented (see also, Rosenbaum, 1998).

According to the timing control view, well-defined motor events are given special status in the control of coordination because such events define the relevant temporal intervals (see also Summers et al., 1989). Coordination may depend critically on the perceptual characteristics of the available motor events. For instance, the finger taps on a response plate might provide sharper timing cues than do the direction reversals during smooth, continuous oscillations of the fingers, hands, or arms. Such differences might account at least in part for the fact that alternate-hand finger tapping can be sustained up to $5 \mathrm{~Hz}$ (i.e., 100 -ms between-hand IRI; Pressing \& Jolley-Rogers, 1997), whereas antiphase finger oscillations typically undergo phase transition at around $2.5 \mathrm{~Hz}$ (e.g., Kelso, 1984). At any event, this example suggests that the timing control of coordination is in principle not limited to slow production rates.

Does timing control selectively operate on the portion of the trajectory that is associated with relevant motor events, or does it include the trajectory as a whole? Similarly, is bimanual coordination achieved by a continuous comparison process between the two limbs or through a relatively discrete process? Although we can not make strong claims here, our working hypothesis is that the control processes associated with representing the temporal events are linked to discrete events. Some light on this issue can be gleaned from studies examining how people learn new patterns of coordination. Zanone and Kelso (1997) were able to train participants to produce bimanual movements with the novel phases of $\phi=0.25$ and 0.75 . However, the novel phase relationships were generally maintained only in the region of maximum flexion of the fingers. For most of the trajectory, the phase delay was close to $\phi=0.0$ or 0.5 . Studies of multifrequency coordination (e.g., one limb oscillating at a given frequency and the other at twice that frequency (Peper, Beek, \& Daffertshofer, in press; Pressing, 1999) have provided similar results. The overall performance generally met the required frequency and phase relationships. At maximum flexion or extension, the two hands coincided every other cycle. However, the dissociation between the trajectories was less than perfect and exhibited tendencies to move in-phase or antiphase over sizable portions of the motion paths (Byblow \& Goodman, 1994; Swinnen, Dounskaia, Walter, \& Serrien, 1997; Walter, Swinnen, Corcos, Pollatou, \& Pan, 1997).

Another aspect of the Zanone and Kelso (1997) study is informative. The participants not only learned the novel phases but exhibited spontaneous transfer of the newly learned coordination (e.g., $\phi=0.25$ ) to the "symmetry partner" (e.g., $\phi=0.75$ ). Such transfer is consistent with the idea that the temporal relationships are represented at an abstract level. What is learned is a particular temporal pattern and is not dependent on the order of the subintervals. Of course, transfer may not be perfect. Learning can occur at multiple levels, some of which may be abstract and others which may be specific to a particular effector or combination of effectors (MacKay, 1982).

This proposal has obvious relationships with a two-level model for motor timing in which, on one level, timing goals are represented by a central timing process, and on another level, movement trajectories are generated by an autonomous motor system in relation with the timing goals (Shaffer, 1992). ${ }^{3}$ It is interesting to note that recent attempts at modeling bimanual rhythmic move- 
ments in the dynamic systems perspective have also espoused a two-level architecture by distinguishing between effector dynamics (the domain of peripheral physical oscillators) and neural dynamics (the domain of coupled central oscillators with timekeeping functions; Peper et al., in press; see also Pressing, 1999). These efforts, like ours, point to the importance of considering the representation of temporal patterns in the control of coordination.

\footnotetext{
${ }^{3}$ Shaffer's (1992) description of motor timing differs from the original Wing-Kristofferson model (Wing \& Kristofferson, 1973) in the interpretation of the motor component.
}

\section{References}

Allan, L. G. (1979). The perception of time. Perception and Psychophysics, 26, 340-354.

Allan, L. G. (1992). The internal clock revisited. In F. Macar, V. Pouthas, \& W. F. Friedman (Eds.), Time, action and cognition: Towards bridging the gap (pp. 191-202). Dordrecht, The Netherlands: Kluwer Academic Publishers.

Baldissera, F., Cavallari, P., \& Civaschi, P. (1982). Preferential coupling between voluntary movements of ipsilateral limbs. Neuroscience Letters, 34, 95-100.

Byblow, W. D., \& Goodman, D. (1994). Performance asymmetries in multifrequency coordination. Human Movement Science, 13, 147-174.

Collier, G. L., \& Wright, C. E. (1995). Temporal rescaling of simple and complex ratios in rhythmic tapping. Journal of Experimental Psychology: Human Perception and Performance, 21, 602-627.

Deutsch, D. (1983). The generation of two isochronous sequences in parallel. Perception and Psychophysics, 34, 331-337.

Essens, P. J. (1986). Hierarchical organization of temporal patterns. Perception and Psychophysics, 40, 69-73.

Essens, P. J., \& Povel, D.-J. (1985). Metrical and nonmetrical representations of temporal patterns. Perception and Psychophysics, 37, 1-7.

Fraisse, P. (1956). Les structures rythmiques [Rhythmic structures]. Louvain, Belgium: Publications Universitaires

Fraisse, P. (1963). The psychology of time. New York: Harper.

Fraisse, P. (1982). Rhythm and tempo. In D. Deutch (Ed.), The psychology of music (pp. 149-180). New York: Academic Press.

Getty, D. J. (1975). Discrimination of short temporal intervals: A comparison of two models. Perception and Psychophysics, 18, 1-8.

Haken, H., Kelso, J. A. S., \& Bunz, H. (1985). A theoretical model of phase transitions in human hand movements. Biological Cybernetics, 51, 347356.

Helmuth, L. L., \& Ivry, R. B. (1996). When two hands are better than one: Reduced timing variability during bimanual movements. Journal of Experimental Psychology: Human Perception and Performance, 22, 278-293.

Ivry, R. B., \& Hazeltine, R. E. (1995). The perception and production of temporal intervals across a range of durations: Evidence for a common timing mechanism. Journal of Experimental Psychology: Human Perception and Performance, 21, 1-12.

Ivry, R. B., \& Keele, S. W. (1989). Timing functions of the cerebellum. Cognitive Neuroscience, 1, 134-150.

Keele, S. W., Pokomy, A., Corcos, D. M., \& Ivry, R. (1985). Do perception and motor production share common timing mechanisms: A correlational analysis. Acta Psychologica, 60, 173-191.

Kelso, J. A. S. (1984). Phase transitions and critical behavior in human bimanual coordination. American Journal of Physiology: Regulatory, Integrative, and Comparative Physiology, 15, R1000-R 1004.

Kelso, J. A. S., Buchanan, J. J., \& Wallace, S. A. (1991). Order parameters for the neural organization of single, multijoint limb movement patterns. Experimental Brain Research, 85, 432-444.
Kelso, J. A. S., Holt, K. G., Rubin, P., \& Kugler, P. N. (1981). Patterns of human interlimb coordination emerge from the properties of non-linear, limit cycle oscillatory processes: Theory and data. Journal of Motor Behavior, 13, 226-261.

Kelso, J. A. S., \& Jeka, J. J. (1992). Symmetry breaking dynamics of human multilimb coordination. Journal of Experimental Psychology: Human Perception and Performance, 18, 645-668.

Killeen, P. R. (1992). Counting the minutes. In F. Macar, V. Pouthas, \& W. F. Friedman (Eds.), Time, action and cognition: Towards bridging the gap (pp. 203-214). Dordrecht, The Netherlands: Kluwer Academic Publishers.

Killeen, P. R., \& Weiss, N. (1987). Optimal timing and the Weber function. Psychological Review, 94, 455-468.

Klapp, S., Hill, M. D., Tyler, J. G., Martin, Z. E., Jagacinski, R. J., \& Jones, M. R. (1985). On marching to two different drummers: Perceptual aspects of the difficulties. Journal of Experimental Psychology: Human Perception and Performance, 11, 814-827.

Kolers, P. A., \& Brewster, J. M. (1985). Rhythms and responses. Journal of Experimental Psychology: Human Perception and Performance, 11, 150-167.

MacKay, D. (1982). The problems of flexibility, fluency, and speed accuracy trade-off in skilled behavior. Psychological Review, 89, 483-506.

Penney, T. B., Gibbon, J., \& Meck, W. H. (2000). Differential effects of auditory and visual signals on clock speed and temporal memory. Journal of Experimental Psychology: Human Perception and Performance, 26, 1770-1787.

Peper, C. E., Beek, P. J., \& Daffertshofer, A. (in press). Considerations regarding a comprehensive model of (poly)rhythmic movement. In P. Desain \& M. L. Windsor (Eds.), Rhythm perception and production. Amsterdam: Swetz \& Zeitlinger.

Peters, M. (1989). The relationship between the variability of intertap intervals and interval duration. Psychological Research, 51, 38-42.

Povel, D.-J. (1981). Internal representation of simple temporal patterns. Journal of Experimental Psychology: Human Perception and Performance, 7, 3-18.

Pressing, J. (1999). The referential dynamics of cognition and action. Psychological Review, 106, 714-747.

Pressing, J., \& Jolley-Rogers, G. (1997). Spectral properties of human cognition and skill. Biological Cybernetics, 76, 339-347.

Rosenbaum, D. A. (1998). Broadcast theory of timing. In D. A. Rosenbaum \& C. E. Collyer (Eds.), Timing of behavior: Neural, psychological, and computational perspectives (pp. 215-235). Cambridge, MA: MIT Press.

Rosenbaum, D. A., \& Patashnik, O. (1980). A mental clock setting process revealed by reaction times. In G. E. Stelmach \& K. Requin (Eds.), Tutorials in motor behavior (pp. 487-499). Amsterdam: North-Holland.

Schmidt, R. C., Carello, C., \& Turvey, M. T. (1990). Phase transitions and critical fluctuations in the visual coordination of rhythmic movements between people. Journal of Experimental Psychology: Human Perception and Performance, 16, 227-247.

Schmidt, R. C., \& Fitzpatrick, P. (1996). Dynamical perspective on motor learning. In $\mathrm{H}$. N. Zelaznik (Ed.), Advances in motor learning and control (pp. 195-223). Champaign, IL: Human Kinetics.

Schöner, G., \& Kelso, J. A. S. (1988). A synergetic theory of environmentally-specified and learned patterns of movement coordination. Biological Cybernetics, 58, 71-80.

Semjen, A., Schulze, H. H., \& Vorberg, D. (2000). Timing precision in continuation and synchronization tapping. Psychological Research, 63, 137-147.

Semjen, A., Vorberg, D., \& Schulze, H. H. (1992). Temporal control in the coordination between repetitive tapping and periodic external stimuli. In C. Auxiette, C. Drake, \& C. Gérard (Eds.), Proceedings of the Fourth International Workshop on Rhythm Perception and Production. Bourges, France: International Workshop on Rhythm Perception and Production. 
Shaffer, L. H. (1992). Rhythm and timing in skill. Psychological Review, $89,109-122$.

Summers, J. J., Bell, R., \& Burns, B. D. (1989). Perceptual and motor factors in the imitation of simple temporal patterns. Psychological Research, 50, 23-27.

Swinnen, S. P., Dounskaia, N., Walter, C. B., \& Serrien, D. (1997). Preferred and induced coordination modes during the acquisition of bimanual movements with 2:1 frequency ratio. Journal of Experimental Psychology: Human Perception and Performance, 23, 1089-1110.

Tuller, B., \& Kelso, J. A. S. (1989). Environmentally specified patterns of movement coordination in normal and split-brain participants. Experimental Brain Research, 75, 306-316.

Vorberg, D., \& Hambuch, R. (1978). On the temporal control of rhythmic performance. In J. Requin (Ed.), Attention and performance VII (pp. 535-555). Hillsdale, NJ: Erlbaum.

Vorberg, D., \& Hambuch, R. (1984). Timing of two-handed rhythmic performance. Annals of the New York Academy of Sciences, 423, 390406.

Vorberg, D., \& Wing, A. (1996). Modeling variability and dependence in timing. In H. Heuer \& S. W. Keele (Eds.), Handbook of perception and action. Vol. 3: Motor Skills (pp. 181-262). London: Academic Press.
Walter, C. B., Corcos, D., \& Swinnen, S. P. (1998). Component variability during bimanual rhythmic movements: Not all harmonic timing ratios are alike. Research Quarterly, 69, 75-81.

Walter, C. B., Swinnen, S. P., Corcos, D. M., Pollatou, E., \& Pan, H.-Y. (1997). Coping with systematic bias during bilateral movement. Psychological Research, 60, 202-213.

Wimmers, R. H., Beek, P. J., \& van Wieringen, P. C. W. (1992). Phase transition in rhythmic tracking movements: A case of unilateral coupling. Human Movement Science, 11, 217-226.

Wing, A. M., Church, R. M., \& Gentner, R. D. (1989). Variability of the timing of responses during repetitive tapping with alternate hands. Psychological Research, 51, 28-37.

Wing, A. M., \& Kristofferson, A. B. (1973). Response delays in the timing of discrete motor responses. Perception \& Psychophysics, 14, 5-12.

Yamanishi, J., Kawato, M., \& Suzuki, R. (1980). Two coupled oscillators as a model for the coordinated finger tapping by both hands. Biological Cybernetics, 37, 219-225.

Zanone, P. G., \& Kelso, J. A. S. (1997). Coordination dynamics of learning and transfer: Collective and component levels. Journal of Experimental Psychology: Human Perception and Performance, 23, 1454-1480.

\section{Appendix}

\section{Derivation of the Response Cycle Variance}

Here we show that when the response cycle is produced as the sum of two shorter subintervals, its standard deviation is less than the standard deviation of the same response cycle when produced without any subdivision.

Consider a sequence of response cycles with mean duration $T$ and standard deviation $\sigma_{T}$ Let $t_{1}$ and $t_{2}$ represent two subintervals of $T$, such that $\left(t_{1}+t_{2}\right)=T, t_{1}=a T$, and $t_{2}=(1-a) \mathrm{T}$, when $0 \leq a \leq 1$. Assumption 1 is

$$
S D\left(t_{1}\right)=a \sigma_{T} \text { and } S D\left(t_{2}\right)=(1-a) \sigma_{T} \quad(\text { Weber's law }),
$$

and Assumption 2 is

$$
\begin{aligned}
& \operatorname{var}\left(t_{1}+t_{2}\right)=\operatorname{var}\left(t_{1}\right)+\operatorname{var}\left(t_{2}\right) \text { (independence) } \\
& \text { and } \operatorname{var}\left(t_{1}\right)=\left(\operatorname{a\sigma _{T}}\right)^{2} ; \operatorname{var}\left(t_{2}\right)=\left[(1-a) \sigma_{T}\right]^{2} .
\end{aligned}
$$

From Assumption 2, it follows that

$$
\operatorname{var}\left(t_{1}+t_{2}\right)=\left(a \sigma_{T}\right)^{2}+\left[(1-a) \sigma_{T}\right]^{2}=\sigma_{T}^{2}\left(2 a^{2}-2 a+1\right) .
$$

If $a=0,1$, then $\operatorname{var}\left(t_{1}+t_{2}\right)=\sigma_{T}^{2}$ (no subdivision), and if $0<a<1$, then $\operatorname{var}\left(t_{1}+t_{2}\right)<\sigma_{T}^{2}$ (subdivision), with $\operatorname{var}\left(t_{1}+t_{2}\right)$ being minimum when $a=0.5\left(t_{1}=t_{2}\right.$, optimal subdivision).

Received June 22, 1999

Revision received May 18, 2000

Accepted May 24, 2000 